\title{
Avdintvm, una nueva forma verbal en celtibérico y sus posibles relaciones paradigmáticas (auzeti, auzanto, auz, auzimei, auzares...) ${ }^{1}$
}

\author{
Carlos JoRdÁn CólERA \\ Universidad de Zaragoza \\ cjordan@unizar.es
}

Recibido: $18-11-2014$

Aceptado: 18-12-2014

\section{RESUMEN}

El Bronce de Novallas es una fragmento escrito en alfabeto latino y lengua celtibérica. En la línea 5 se lee audintum. El autor propone analizarla como el genitivo plural del participio de presente de un verbo, cuya base proto-celtibérica sería $* h_{2} e w-d^{h} e h_{1}-{ }^{*} h_{2} e w-d^{h} h_{1^{-}}-0$, simplemente, $* a u d^{h} e h_{1}-*^{*} a u d^{h} h_{1^{-}}$. Su significado pertenecería a la esfera de 'conceder, otorgar, donar'. También pertenecerían a este paradigma otras formas encontradas en el corpus celtibérico: auzeti, auzanto, auz (¿auzati?) y auzimei. Quizá también esté relacionado etimológicamente auzares.

Palabras clave: Bronce de Novallas, audintum, epigrafía celtibérica, lengua celtibérica, verbo celtibérico.

\begin{abstract}
The Novallas bronze plaque is a fragmentary text written in Latin alphabet and Celtiberian language whose fifth line contains the word audintum. The author proposes to analyse it as the genitive plural of the present participle of a verb. The proto-Celtiberian base would be $* h_{2} e w-d^{h} e h_{1}^{-/ *} h_{2} e w-d^{h} h_{I^{-}}$or, simply, $* a u d^{h} e h_{1}^{-/ *} a u d^{h} h_{1^{-}}$. His meaning would be 'to give, to grant, to donate'. The Celtiberian terms auzeti, auzanto, auz (auzati?) and auzimei may be etymologically related to this stem and possibly so is auzares.
\end{abstract}

Keywords: Novallas bronce plaque, audintum, Celtiberian epigraphy, Celtiberian language, Celtiberian verb.

\section{SUMARIO}

PRESENTACIÓN - audintum - auzeti [K.1.1, A-10] - auzanto [K.1.3, 02] - auz [K.0.8], ¿[K.5.1] y [K.2.1]? auzimei - auzares [K.0.14, B-2, -6] - ReCAPITULACIÓN - NotA FINAL - BiblografíA

${ }^{1}$ Este trabajo ha sido realizado en el marco del Proyecto de Investigación «El nacimiento de las culturas epigráficas en el Occidente mediterráneo (II-I a. E.)», FFI2012-36069-C03-03, dirigido por el Dr. F. Beltrán Lloris, de la Universidad de Zaragoza. 


\section{PRESENTACIÓN}

En el XI Coloquio sobre Lenguas y Culturas Prerromanas de la Península Ibérica, celebrado en Valencia (24-27 de octubre de 2012), realizamos un primer avance del que hemos denominado Bronce de Novallas. ${ }^{2}$ Es un fragmento de una placa de bronce, hallado en las cercanías de Novallas, provincia de Zaragoza, con unas medidas máximas de $18,1 \times 22,5 \times 0,2 \mathrm{~cm}$. En ella pueden leerse restos de diez líneas de texto, escrito en alfabeto latino y, en principio, lengua celtibérica. En la línea 5 aparece la palabra audintum. En concreto se lee:

\section{[---]am·audintum·odas·publicuș[..]}

\section{audintum}

El contexto sintáctico, debido a la fragmentariedad del texto, no ayuda mucho a su análisis. La palabra anterior a audintum termina en -am. Pocas cosas podemos decir con absoluta certeza de ella, excepto que apunta a un acusativo singular de un tema en $-a$. En cuanto a la secuencia siguiente, odas publicus, ya adelantamos en su momento ${ }^{3}$ su análisis como un sintagma en acusativo del plural de un tema en dental (odas) y de un tema en -o (publicus), palabra préstamo del latín. Lo que no estamos en disposición de confirmar es su dependencia sintáctica. ${ }^{4}$

Basaremos, pues, nuestro análisis, sobre todo, en su aspecto morfo-etimológico. La forma parece un genitivo del plural de un tema en -nt-, procedente de *audi-nt-om. Proponemos analizarla como el genitivo del plural del participio de presente de un verbo que, según creemos, ya está testimoniado en celtibérico en, al menos, dos formas: auzeti [K.1.1, A-10] y auzanto [K.1.3, 02]. Es posible que también pertenezca al mismo paradigma auz [K.0.8, -2] y [K.5.1]; auzạ[---] [K.2.1]; y quizá auzares [K.0.14, B-2, -6]. Relacionado también etimológicamente se hallaría auzimei (Plomo de La Manchuela, 1. 4), en forma de sustantivo. Como es bien sabido, no existe unanimidad en la etimología y morfología de todas estas formas. ${ }^{5}$ En este trabajo iremos seleccionando los datos que creamos oportunos para nuestra exposición.

Schumacher ${ }^{6}$ ha propuesto para la base verbal celtibérica auz- (aud- en su transcripción), siguiendo la senda que ya marcara Untermann en su momento, ${ }^{7}$ una raíz secundaria $* h$ e $w d^{h}$-, que, a su juicio, puede dar explicación del tema de presente del irl. ant. odaid*, oid 'dejar, prestar' ('leihen') y sería la responsable de la base germánica *aud- 'conceder, otorgar, donar', isl.ant. auðenn 'verliehen', ing. ant. ēaden 'gegeben, gewährt' y saj. ant. ōdan 'geschenkt'. Esta base procedería de una secuencia

${ }^{2}$ Beltrán Et ALII (2013). Las indicaciones tipo [K.1.1] hacen referencia Untermann (1997).

${ }^{3}$ BeLtrán ET ALII (2013: 623).

${ }^{4}$ Para lo cual vid. JORDÁn (2014a: 339-340).

${ }_{5}^{5} \mathrm{Vid}$. para las diferentes propuestas, por ejemplo, WodTKo (2000: ss.uu.), hasta el año 2000; JoRDÁN (2004: 141 y ss.); de Bernardo (2000), (2007a), (2007b), y (2010); Prósper en Villar y Prósper (2005), y (2007) y (2008); Rubio (2013).

${ }^{6}$ SCHUMACHER (2004: 728-737).

${ }^{7}$ UnTERMAnN (1997: 412, §665), en donde hablaba de la raíz *ə $2 u d^{h}$ - 'conceder, donar'. 
anterior $* h_{2} e w-d^{h} h_{1}-$, en definitiva, un compuesto $* h_{2} e w-d^{h} e h_{1}-{ }^{8}$ Este compuesto tendría su reflejo en el verbo hetita pēhutemi 'llevar (a), conducir (a), ejecutar, realizar, llevar a cabo', procedente de ${ }^{*} h_{1}$ poi- $h_{2} \mathrm{ou}-d^{h} e h_{1}-m i{ }^{9}{ }^{9}$ Por lo que se refiere al celtibérico, el autor se centra sobre todo en el análisis de auzeti y auzanto y deja a un lado auz por ser posiblemente una abreviatura; auza[---] por problemas de lectura (no se sabe si la vocal final forma parte de la secuencia anterior o no); y para auzares no termina de dar un análisis definitivo. ${ }^{10}$

Pues bien, atendiendo a la forma audintum y quizá auzimei, creemos que efectivamente la base de la que debemos partir para establecer la conexión entre todas estas formas es * $h_{2} e w-d^{h} e h_{1}-/ * h_{2} e w-d^{h} h_{1}-$, en donde todavía «se tenía conciencia morfológica» del elemento radical fundamental $* d^{h} e h_{l}-/ d^{h} h_{l^{-}}$.

También podría pensarse, como indicara Rubio, ${ }^{11}$ que el elemento inicial au- es el preverbio $a u$-, que tiene buen refrendo en lenguas indoeuropeas, como tal en latín, tipo aufero, aufugio, o como preposición en irlandés antiguo ólúa $<a u$. Este autor, sin embargo, posteriormente ${ }^{12}$ prefirió por motivos semánticos establecer la relación etimológica de las distintas formas indicadas sobre la raíz *deh - 'dar'. En su último trabajo al respecto, ${ }^{13}$ parece dejar la puerta abierta de nuevo a la raíz $* d^{h} e h_{l^{-}}$, pues da como posible paradigma verbal celtibérico: auz(a)- $[0$ au-z(a)- $=i \mathbf{z i z}-/ \mathbf{t a}-$, -za- con preverbio au-?] 'dar, poner'.

La proto-forma del nominativo singular masculino del participio en cuestión sería, según nuestra propuesta, *au-d $h_{l}$-ént-s (a partir de ahora solucionamos ya la secuencia * $h_{2} e w$ - como $\left.a u-\right)$, según el patrón histerodinámico que indicara $\mathrm{Rix}^{14}, c f$. griego $\tau \imath \theta \varepsilon i ́ c$ procedente en primera instancia de $* d^{h} i-d^{h} h_{-}$-ént-s. Todavía no tenemos datos seguros de cómo pudo evolucionar la parte final del reconstructo en celtibérico. En efecto, en este punto, como en la mayoría de la gramática celtibérica, estamos bastante huérfanos. Las palabras que se han considerado temas en -nt- son:

1. tirikantam [K.1.1, A1]. Hasta la aparición del cuarto bronce de Contrebia Belaisca, se pensaba que esta palabra era el acusativo singular de un tema en $-a$, excepción hecha de Eska, ${ }^{15}$ quien lo consideraba un tema en -nt-. Para este autor era el acusativo singular femenino, procedente de *-nt-m. La asignación de dicho género era argumento suficiente, a juicio de Wodtko, ${ }^{16}$ para dudar de este análisis, pues lo espera-

${ }^{8}$ Para * $h_{2} e w$ - 'disfrutar' vid. Rix ET ALII (2001: 274), Pokorny (1948-1959: 77-78); para * $d^{h} e h_{1}$ - 'colocar, poner, establecer, producir, hacer', vid. RIX ET ALII (ibid.: 136-138), POKORNY (ibid.: 235-239).

${ }^{9}$ Siguiendo las indicaciones de KloeKhorst (2007: s.u. pehute ${ }^{z i} /$ pēhut-).

${ }^{10}$ Schumacher (2004: 759-760).

${ }^{11}$ Rubio (1996: 185).

${ }^{12}$ Rubio (1999a: 108 y ss.); (1999b: 622 y 626-627).

${ }^{13}$ Rubio (2013: 710). En este paradigma incluye: el teórico auzu [K.5.1] (lectura alternativa auz) que analiza como $1 .^{\mathrm{a}}$ sig. pres. ind.; auzeti [K.1.1, A10], 3. ${ }^{\mathrm{a}} \mathrm{sg}$. pres.subj.; auzanto [K.1.3, 01], 3. ${ }^{\mathrm{a}} \mathrm{pl}$. pretérito > imperativo medio; auzares [K.12.1] i $3 .{ }^{a}$ pl. perfecto? i $3 .{ }^{a} \mathrm{sg}$. pasiva + enclítico es?; auz [K.0.8], forma abreviada, o $\measuredangle$ bien /auūð/ = galo AVOT?; y auza [K.2.1], forma fragmentaria, $\iota^{*}$ auza[az]?

${ }^{14} \operatorname{RIX}(1976: 233-234, \S 256)$.

${ }^{15}$ ESKA (1996).

${ }^{16}$ WodTKo (2000: s.u. tirikantam). 
do en el femenino de un tema en -nt- sería una forma $-n t-\bar{\imath}<*-n t-i h_{2}$. A decir verdad, en ningún momento Eska indica que sea una forma adjetiva, al menos en ese artículo, por lo tanto es una suposición de Wodtko, quien tampoco tuvo en cuenta para el género la aparente concordancia con berkunetakam.

Con la aparición de tirikantam y tirikantos en las 11.1 y 3 de [BBIV], quedaba ya fuera de dudas de que era un tema en $-n t$-, según se indica en Villar et alii. ${ }^{17} \mathrm{Se}$ guramente estamos hablando de dos casos de un apelativo, cuya primera forma es el acusativo con el tratamiento de la nasal final tal y como lo había previsto Eska y con la posterior frontalización en las lenguas celtas insulares, siguiendo a McCone; ${ }^{18}$ la segunda forma es el genitivo del singular. El género debe ser femenino por la concordancia indicada en [K.1.3] y por la más que probable de [BBIV] [---] tam tirikantam. No éramos capaces, entonces, de determinar el aspecto de su correspondiente nominativo del singular.

Prósper ${ }^{19}$ propone que es el acusativo singular del participio de presente de $* d^{h} r g$ $n t-o * d^{h} r \hat{g}^{h}-n t-$ 'que abarca, contiene o recoge dentro de sí', ${ }^{20}$ con un grado cero del morfo por extensión desde, por ejemplo, el genitivo singular tirikantos $<*$ - $d^{h} r g$-ngt-os $0 * d^{h} r \hat{g}^{h}-n t-o s$, que aparece en [BBIV]. De manera que tirikantam $<{ }^{*} d^{h} r g-n t-m$ o $* d^{h} r \hat{g}^{h}-n t-m$. No indica cómo sería el nominativo. Este análisis no es, por supuesto, definitivo. ${ }^{21}$

2. Otra palabra para la que se ha propuesto esta clasificación es la que aparece testimoniada como steniotes [K.17.1], stenion+ [K.1.3, IV-2] y Stenionte [K.11.1]. Aquí, los testimonios no son definitivos para la clasificación como tema en - nt- y cabe incluso la posibilidad de que fuese un tema en $-i{ }^{22}$ De ser, no obstante, un tema en - nt(opción por la que nos inclinamos) parece que a lo largo del paradigma se extendió el alomorfo -ont-.

3. Luján ${ }^{23}$ ha propuesto la existencia de otro tema en -nt-. Se trataría de la forma melmaz que aparece en el plomo de la Manchuela (1. 6), también denominado plomo de Iniesta. Procedería de una base celtibérica *melmant-, con un sufijo originario *-ment-/-mnt-, que con desinencia $-s$, al igual que ocurre en estos temas en irlandés y en britónico, quedaría como *melmants. En celtibérico la evolución de la forma habría sido *melmants $>*$ melmanz $>$ melmaz.

Pues bien, si el sufijo es el indicado, la vocal $-a$ - originaria de *melmant- procede, evidentemente, del morfo en grado cero y tiene que ser breve. La -a- final, de melmaz, ya podría ser larga como consecuencia de un alargamiento compensatorio al simplificarse el grupo final ${ }_{-}-n t s>-n z>-z$. Si esto último que decimos fuera cierto, aplicado

${ }^{17}$ Villar et aliI (2001: 104-107). De Bernardo (2010: 125) sigue pensando que tirikantam es un tema en - $a$, de lo que deducimos que tirikantos debe pertenecer a otro paradigma.

${ }^{18}$ MCCone (1996: 48-51, 54-59 y 70-79).

${ }^{19}$ Prósper (2008: 20).

${ }^{20}$ Pokorny (1948-1959: 212 y 254) s.uu. $* d e r g^{h}-\mathrm{y} * d^{h}$ ere $\hat{g}^{h}$ - respectivamente.

${ }^{21}$ Para otras interpretaciones, vid. WоDTKо (2000: s.u. tirikantam).

${ }^{22} \mathrm{Vid}$. para este asunto VILLAR ET ALII (2001: 104-107).

${ }^{23}$ LUJÁN (2009: 703-704). 
al caso que nos ocupa y ajustándonos a la ortodoxia en la formación del participio de presente, se podría suponer una evolución $* a u-d^{h} h_{l}$-ent-s $>* a u z e n z>* a u z \bar{e} z>$ *auzizz, en donde aparecería una vocal $\bar{l}$. Sin embargo hay que confirmar primero que en melmaz no aparece la nasal -n- porque ha desaparecido realmente y no se trata de una omisión gráfica (tipo sekotiaz [A.77] por *sekontiaz). Obsérvese en este sentido que el anteriormente citado stenion+ [K.1.3, IV-2] parece ocupar sintácticamente el lugar de un nominativo. Si esto es así, la vocal -o- en sílaba final no había sufrido alargamiento, pues tendría que haber aparecido como -u-. ¿Hay que suponer, por lo tanto, un nominativo *stenionz y, correspondientemente, *melmanz?

Una alternativa para la aparición de una $-\overline{1}-$, independientemente de lo que pasase con la parte final -nts, es pensar que este participio de presente se formó por analogía con el tema del presente de indicativo, que presentaba al menos en singular el grado pleno de la raíz, es decir, ${ }^{*} a u-d^{h} e h_{l}$-ent-s. Esta vocal se extendería a todo el paradigma siguiendo un proceso de nivelación analógica, fenómeno bastante común a este tipo de paradigmas, como demuestran la mayoría de las lenguas indoeuropeas. Así aparecería un genitivo singular *au-d heh ${ }_{1}$-nt-os $>*$ audēntos $>*$ audintos, de donde un plural *au$d^{\text {heh }}$-nt-ōm $>$ *audēntōm $>*$ audīntum.

Si nos detenemos en el aspecto de la vocal, es porque al esperado nominativo *au$d^{h} h_{l}$-ént-s le habría correspondido un genitivo *au- $d^{h} h_{l}-n t$ t-os, en singular, *au- $d^{h} h_{1}$ - not$\bar{o} m$, en plural, que habrían desembocado en *auđantos $\mathrm{y} *$ auđantum, correspondientemente, lejos de una forma *audintum, que es la que refleja audintum, según creemos. Personalmente, todavía no somos capaces de determinar el comportamiento del acento en celtibérico, como para proponer una metafonía de $\breve{a}$ en $\breve{l} .{ }^{24}$

\section{auzeti [K.1.1, A-10]}

Sobre auzeti Schumacher ${ }^{25}$ indica, como ya habían hecho antes otros autores ${ }^{26}$ que por el contexto en el que se inserta, ...ios uramtiomue auzeti aratimue tekametam tatuz..., parece que lo más apropiado es pensar que estamos ante un subjuntivo. En efecto, antecede a una forma verbal en imperativo, tatuz, y, por lo tanto, no sería extraño una forma de carácter modal. Si pensamos en la raíz y tema verbal propuestos, la única solución es pensar en una 3. ${ }^{a}$ persona del singular del presente de «subjuntivo temático», voz activa, *au-d $h_{l} h_{l}-e-t i$, con el tema conformado sobre el del aoristo en grado cero. Esto es, un subjuntivo temático de un correspondiente indicativo atemático, que tal y como hemos avanzado aparecería en grado pleno, *au-dh $e h_{-}-t i>* a u-d^{h} \bar{e}-t i>$ $* a u$-đt-ti, que se grafiaría < auziti> en signario paleohispánico, $<$ audit $(i)>$ en alfabeto

${ }^{24} \mathrm{Ni}$ siquiera aplicando la reconstrucción del acento proparoxítono y sus efectos que supone DE BERNARDO (2007: 156-159), para el celta hispano. El contexto que más se acercaría sería el de la reducción de la sílaba postónica (*áuđantos $>$ *áuđintos y *áuđantum $>*$ *ađintum), pero los ejemplos que aporta la autora contienen bien $\breve{e}$, bien $\breve{o}$, bien $\breve{u}$ postónicas que se convierten en una vocal débil [ə] que acaba siendo grafiada por $a$, en alfabeto latino, y a/ai en signario paleohispánico en su variedad celtibérica. Desgraciadamente no nos dice qué pasa con una $a$ postónica originaria. ¿Llegamos también a una [ə] que se grafía con $a$ ? Entonces, poco hemos adelantado.

${ }^{25}$ SCHUMACHER (2004: 734-737).

${ }^{26}$ Para esto vid. Wodtкo (2000: s.u. auðeti). 
latino. Si se hubiese conformado también sobre el grado cero, entonces el aspecto sería $* a u-d^{h} h_{1}-t i>* a u-d{ }^{h} \breve{a}-t i>* a u-đ \breve{a}-t i,<$ auzati $>$ en signario paleohispánico, <audat $(i)>$ en alfabeto latino.

Consideraciones contextuales, nos obligan a preferir la raíz en grado pleno para el presente de indicativo y la raíz en grado cero para formas de aoristo, sobre cuya posible existencia, vamos a hablar a continuación.

La posibilidad de que auzeti sea una $3 .{ }^{\mathrm{a}}$ persona del singular del aoristo de subjuntivo, radical temático de una base * $a u d h_{3^{-}}$, de la raíz * $d h_{3^{-}}$'dar', como ha estado proponiendo $\mathrm{Rubio}^{27}$ presenta un pequeño inconveniente, a nuestro juicio: la evolución de la laringal. Obsérvese que el autor siempre se ve en la necesidad de reconstruirla entre paréntesis: *-d(h)-e-ti /*-d(ə)-e-ti. ¿Por qué no deja huella (*auzoti, según la ortodoxia) y sí lo hace en auzanto, que restituye como ${ }^{*}-d \partial_{3}-n t o-?$ Resulta un comportamiento un tanto sospechoso.

\section{auzanto [K.1.3, 02]}

Como indica Schumacher ${ }^{28}$ en auzanto la terminación -nto, además de enviar a la voz media, nos está indicando un tiempo de pasado. El texto en que aparece es el siguiente:

\section{risatioka.lestera.ia.tarakuai.nouiza.auzanto / eskeninum.taniokakue.soisum.albana}

Parece el verbo de una oración de relativo, introducida por ia. El tipo de inscripción ante el que nos encontramos, listado de nombres, no aconsejan pensar en un imperfecto. Estos hechos apuntan, mejor, a una forma de aoristo radical atemático de indicativo, con un significado final del tipo «wurden gewährt, wurden gegeben» («han sido concedidas / fueron concedidas / se han concedido / se concedieron»), como ya indicara Untermann. ${ }^{29}$ La protoforma sería, por lo tanto, *au-dh $h_{l}-$ nto.

\section{auz [K.0.8], ¿[K.5.1] y [K.2.1]?}

La secuencia auz [K.0.8] y [K.5.1] plantea, como es bien sabido, un serio problema. En [K.0.8] parece efectivamente una secuencia aislada, si hay que leer, como estamos convencidos, el documento letontu auz soz. Untermann ${ }^{30}$ proponía restituir una tercera persona de pretérito *auzez $<* a u d^{h}$-e- $t$, cuya forma de presente sería el ya comentado auzeti. Ahora bien, analizada auzeti tal y como hemos hecho, cabe un análisis de auz como forma verbal completa si pensamos de nuevo en un aoristo radical atemático, pero ahora en voz activa. Esto nos lleva a proponer una forma originaria *au-dh $h_{t}-t$, cuyo grupo consonántico final acabaría simplificando en lo que en signario celtibérico acabó por consignarse mediante una sigma, seguramente algo

\footnotetext{
${ }^{27}$ Rubio (1996: 185); (1999a: 108); (1999: 626-627).

${ }^{28}$ SCHUMACHER (2004: 735).

${ }^{29}$ UNTERMANN (1987: 585).

${ }^{30}$ UNTERMANN (1987: 412) y los correspondientes comentarios a las piezas.
} 
parecido a una fricativa interdental sorda $[\theta] .{ }^{31}$ Ahora bien, no podemos soslayar que entonces debemos aceptar una no vocalización de la laringal interconsonántica, que tendría que haber desembocado en *auđăt $>$ *auđat. Entonces es legítimo plantearse, siguiendo una vez más la senda abierta por Untermann, que auz puede ser la expresión escrita abreviada de *auzaz que se hallaría de forma casi plena en [K.2.1] [---] ẹtukenosauzạ[---], según nuestra lectura (autopsia 15.03.07). En efecto, una primera segmentación permite aislar [---] ẹtukenos, casi con seguridad retukenos. A continuación está claro que se lee la secuencia auz seguida de a y a partir de allí ya no sabemos qué pudo haber escrito, sencillamente porque ya no hay más pieza.

Prósper ${ }^{32}$ llevaba a cabo la restitución auza[res], para así coincidir con la palabra auzares que aparece en el Bronce Res [K.0.14] dos veces. El carácter especulativo de la propuesta parece reconocerlo la misma autora en 2006, en donde considera preferible interpretar esta pieza como [r]etukenos auz a[---bos], con una estructura sujeto-verbo-coda, paralela a la de [K.5.1] beskuauzuetikubos $\rightarrow$ «Bescón $(=$ besku) ha hecho/firmado (= auz). Para (la familia de) los véticos' (= uetikubos)». La autora equiparaba la forma auz a la gala avot, cosa que considerábamos acertada en su momento, pero que en la actualidad ya no podemos aceptar. Ambas formas serían, para Prósper, la tercera persona de un aoristo radical atemático, con preverbio *hew-, y contempla partir de una protoforma *au-ūt $\left(0^{*} a u-w \bar{u} t\right)$, que en galo da [awột] (o [auwột]), con disimilación; y en celtibérico [awūz] (o [auwūz]), sin ella. A modo de posibilidad etimológica propone la raíz * (h)weh- 'tejer', cuyo tema en aoristo sería *(h)weh- para el singular y *(h)uh- para el plural. Habría una generalización a todo el paradigma de este tema. auz no tendría relación con auzeti y auzanto, que analiza como una $3 .^{\text {a }}$ singular y plural del subjuntivo de aoristo sigmático de la raíz *h, ew- "exigir, requerir, aceptar', ${ }^{*} h_{2} e w-s-e-t i$ y $* h_{2} e w-s-n t o .{ }^{33}$ La sigma del signario paleohispánico, transcrita -z-, estaría marcando el resultado de la silbante originaria intervocálica, que sería la marca de aoristo. ${ }^{34}$ Tampoco podemos aceptar ahora esta propuesta para auzeti y auzanto, pues ya no compartimos el tratamiento de la silbante originaria intervocálica que propone Prósper, more Villar. Creemos que debería haberse mantenido, more Untermann, *auseti y *ausanto (escritas con san en signario paleohispánico). A día de hoy no encontramos ninguna etimología incontestable para mantener la propuesta originaria de Villar.

De Bernardo ${ }^{35}$ ha propuesto segmentar el mensaje de [K.5.1] besku auzu etikubos y lo traduce como 'Yo pesco, yo extraigo (= auzu) para las X (= etikubos)' o 'De Y (= besku) yo extraigo (= auzu) para las X (= etikubos)'. Es decir, que estaríamos ante un objeto, un oinochoe, parlante. Además de la primera persona auzu, aparecería otra primera besku que sería un préstamo del latín, de ahí la traducción 'Yo pesco'. Aunque no acaban ahí las posibilidades propuestas por la autora, porque también podría

${ }^{31}$ Sobre esta cuestión vid. JORDÁN (e.p.).

${ }^{32}$ Prósper en Villar y Prósper (2005: 320).

${ }^{33}$ Prósper (2008: 73).

${ }^{34}$ LAMBERT (2002: 32-35) consideraba el galo avot una formación de perfecto originaria con preverbio separativo *au-wō $d^{h}$-ti, de una raíz *wed $d^{h}$ - 'conducir', de donde un significado 'ejecutar, llevar a término'.

${ }^{35}$ De Bernardo (2007a). 
tratarse de un tema en - $u$ neutro, bien besku bien pesku (de nuevo préstamo) que sería el objeto directo de auzu, de donde 'Yo extraigo algo'. Y como última posibilidad podría ser un ablativo de un tema en -o, bien beskom o peskom, que expresaría una modificación local del verbo, de donde la traducción 'De X, yo extraigo...'. La forma etikubos parece que es morfológicamente transparente como dativo plural, bien de un genónimo que expresaría el nombre del grupo familiar al que va destinado el oinochoe ('para los Éticos'), bien un nombre común o de acción. Da como ejemplo [edikubos] 'for the meals/dinners', sin percatarse de que la dental sonora originaria intervocálica debería haber sido grafiada con sigma en el signario paleohispánico, según se acepta de manera general, y aparecer la palabra como *ezikubos.

La división del texto es viable, pero encontramos problemas para aceptar esta interpretación del grafito por distintas razones. Ciñéndonos a dos aspectos lingüísticos:

1. Nos resulta difícil aceptar un préstamo latino para besku, sobre todo como primera persona del verbo 'pescar', pues hay que partir de una forma latina piscor (deponente) y no llegamos a entender bien el motivo del préstamo.

2. La propuesta etimológica de auzu, a partir de la raíz $* h_{2} e w s-$, cuyo significado es 'sacar, extraer', $c f$. latín haurio, no nos parece adecuada por el tratamiento de ese originaria intervocálica, según acabamos de exponer unas líneas arriba. A esto hay que añadir que tampoco nos parece ajustada por razones interpretativas y mucho menos históricas, si se acepta la visión de la autora sobre la extracción y reparto de aguas en época celtibérica. Pero no vamos a entrar en esta cuestión y nos vamos a centrar en lo estrictamente lingüístico. ${ }^{36}$

En efecto, de Bernardo considera que auzeti, auzanto y auza(to)/auza(ti) (restitución de la forma incompleta auzạ[---] de [K.2.1], según la autora) tienen la misma etimología que auzu, con los siguientes análisis morfológicos:

auzeti $<* a u s-e-t i, 3{ }^{a}$ p. sg. del presente de indicativo, voz activa; auzanto $<*$ aus $-\bar{a}-n t o$ es la $3{ }^{a}$ p. sg. de presente de subjuntivo de la voz media; auza(to)/auza(ti) $<* a u s-\bar{a}-t o /-t i$ es la correspondiente $3 .^{\text {a }}$ del singular, bien en voz media, bien en activa.

Esta relación, con el mismo análisis morfológico, se puede establecer entre estas formas partiendo de $* a u d^{h} e_{l^{-}}$. Esto supondría aceptar que el celtibérico habría operado de igual manera o parecida al latín, si es que verbos como reddĕre y, más claramente, abděre, conděre, son formaciones con preverbio de la misma raíz, en donde se habría dado una tematización del tema débil (con el vocalismo en grado cero) ${ }^{37}$ Sin perder de vista, no obstante, que también podría estar implicada la raíz *deh $h^{-}$'dar'.

Esta atractiva relación paradigmática presenta dos pequeños inconvenientes, a nuestro juicio:

${ }^{36} C f$. últimamente de Bernardo (2013: 645-649). Los paralelos antiguos que propone la autora no son iguales ni parecidos a lo que ella supone que pasaba en Contrebia Belaisca. Sobre los paralelos modernos, preferimos no comentar nada.

${ }^{37}$ Tal y como se indica en RIX ET ALII (2001: 136-138). 
1. Por un lado, aparecería un indicativo, auzeti, en donde esperamos sintácticamente un subjuntivo; un subjuntivo, auza(to)/auza(ti), donde esperamos un indicativo; y habría que explicar el subjuntivo, auzanto, de la oración de relativo. De momento podríamos salvar la situación argumentando nuestro desconocimiento de la sintaxis celtibérica.

2. Por otro lado, audintum quedaría fuera de juego, pues esta forma participial difícilmente se puede explicar como perteneciente a un paradigma de un verbo temático. La solución podría pasar por pensar que estamos ante dos paradigmas diferentes: el de auzu, auzeti, auzanto, auza(to)/auze(ti) y el de audintum. La otra es esperar a que se confirme la existencia de auzu como una palabra real.

\section{auzimei}

Los editores del plomo de La Manchuela o plomo de Iniesta, Lorrio y Velaza, ${ }^{38}$ apuntaban al carácter verbal de la palabra auzimei (línea 4) y su relación con auzeti y auzanto. Presentaban como paralelo para el final tiatumei $[K .3 .3,-2]$. Nos topamos aquí de nuevo con un problema de lectura, precisamente en el lugar de la nasal, que más bien parece una - $n$-, de donde tiatunei (autopsia 23.11.05).

Aunque se podría retorcer morfológicamente auzimei hasta considerarla una forma personal (primera persona del plural) no parece muy factible, además de por la morfología en sí, por el contexto, donde no terminamos de ver la aparición de esa persona (aunque esto puede deberse de nuevo a nuestro desconocimiento de la lengua celtibérica) y porque parece concordar con la palabra anterior, rouzunei.

Prósper $^{39}$ ve en auzimei un topónimo o un antropónimo *Audimo- o *Ausimo-. El antropónimo, en locativo, tendría su explicación pensando en una expresión tipo español «donde Martín», francés chez Martin, con lo que auzimei habría que entenderlo como «en la propiedad de Ausimo». El locativo con un topónimo no precisa de más explicación. También podría tratarse de un apelativo que indica la localización de algo. No concordaría con rouzunei, que analiza como un dativo de un tema en nasal de un antropónimo *roudū, equivalente al latino Rufō. Deja la puerta abierta también a un locativo de un topónimo de tema en - $o$, *rouzunos. A juzgar por las interpretaciones que lleva a cabo del comienzo del documento, parece preferir que es un antropónimo: «...para Roudón, en Audimo».

De Bernardo, ${ }^{40}$ por su parte avanzaba de manera tentativa el siguiente doble análisis de auzimei: bien un nombre verbal en $-m$ - relacionado con los verbos auzu y auzeti, con la etimología ya indicada; bien el dativo de un nomen agentis del mismo tema verbal concordando con la palabra anterior rouzunei (si resulta ser el dativo de un idiónimo en nasal, ${ }^{*}$ roud $\left.{ }^{h} y \bar{o}\right)$.

También nosotros pensamos que existe una relación etimológica entre las probables formas verbales celtibéricas analizadas que comienzan por auz- y auzimei. Proponemos por ello también una posible doble etimología y análisis morfológico:

\footnotetext{
${ }^{38}$ Lorrio y Velaza (2005: 1038).

${ }^{39}$ PRÓSPER (2007: 31-32).

${ }^{40}$ De Bernardo (2007: 65, n. 63).
} 
1. Considerar un nominativo *au-dheh-mi-s $>*$ audimis, con un sufijo -mi-, en absoluto extraño en el panorama indoeuropeo, en cuyo paradigma auzimei sólo podría entenderse como dativo. Una formación $* d^{h} e_{1}-m i$ - es la responsable del sustantivo femenino en avéstico dāmi- 'creación', también adjetivo 'creador, -a' ${ }^{41}$

2. Pensar en un nominativo *au- $d^{h}$ eh - mo-s $>$ *audimos, con el sufijo -mo-, menos abundante, con lo que auzimei sólo podría ser su correspondiente locativo. ${ }^{42}$

La utilización de una secuencia igual, sin el primer miembro de compuesto, había sido propuesta por Prósper ${ }^{43}$ para temei-ue [K.1.1, A-8] $<* d^{h} e h_{-}-m o-/-m i-$. Sin embargo, es bastante difícil de sostener, pues el resultado habría sido *tī-mo-/-mi-, con el cierre esperado de $\bar{e}$, a no ser que obviamente supongamos que no se ha producido. El correspondiente grado cero habría dado *ta-mo-/-mi-.

\section{auzares [K.0.14, B-2, -6]}

Casi simultáneamente, Rubio y Untermann plantearon la posibilidad de que auzares estuviese relacionado morfo-etimológicamente con la serie auzeti, auzanto, auz. El problema es determinar cómo. También ha habido autores que consideran que no hay relación etimológica entre estas formas. ${ }^{44}$

Untermann ${ }^{45}$ se decantaba por analizar auzares como el nominativo plural de un sustantivo verbal que podía ser utilizado como forma finita.

Rubio ${ }^{46}$ proponía ver en auzares una tercera persona del plural de perfecto, con una desinencia *-r $(s)$, que podría detectarse también en kombalkores [K.1.1, A-11], y que tendría su paralelo, aunque no exacto, en la familia indo-irania. Suponemos (pues no lo indica expresamente) que la forma originaria sería *audh $-r(s)$. Aquí la laringal sí se transformaría en lo esperado, de forma que se llegaría a auzar-. No queda claro, sin embargo, el origen de la vocal -e-.

Precisamente es la terminación -es el mayor obstáculo para considerar auzares como una forma personal del mismo paradigma que auzeti y auzanto. No hay mayor problema en detectar en las lenguas indoeuropeas una marca $*_{-} r$ como desinencia. Como es bien sabido, la podemos encontrar marcando medio-pasividad, impersonalidad, formas de pretérito y de perfecto. En nuestro caso, en concreto, se podría pensar

${ }^{41}$ Pokorny (1948-1959: 238).

${ }^{42}$ Para los sufijos - $m i$ - y -mo- en irl. ant. en el contexto de las lenguas celtas e indoeuropeas $c f$. DE BERNARDO (1999: 244-246).

${ }^{43}$ PRÓSPER (2008: 66).

${ }^{44}$ Para lo cual vid. Wodtкo (2000: s.u. auðares), hasta la fecha de su publicación. Prósper en VILlAR y PRÓSPer (2005: 205-205, 301 y 326) analiza esta forma como una tercera persona de un aoristo sigmático *aud(i)-ad-rexst 'ha depositado'; lo mismo que esozeres, aunque como forma corrupta de un esauzares... o bien una forma muy cercana. Motivos paleo-epigráficos no nos permiten aceptar sin más esta propuesta, vid. JORDÁN (2005-2006: 482-484). En concreto, nos preguntábamos por qué no se indicaba la dental final, como podría estar sucediendo en unameiste, palabra que aparece en la tercera línea de la teórica cara A del Bronce Res y que nosotros leíamos entonces como teameiste. A la luz de nuevo material fotográfico conseguido, consideramos que esta última lectura teameiste debe ser rechazada y proponemos lameiste, vid. JORDÁN (2014: 308-309).

${ }^{45}$ UnTERMANN (1997: 409 y 561).

${ }^{46}$ Rubio (1996: 185 y 190-191). 
en una proto-forma * $a u d^{h} h_{1}-r$ responsable de auzar-. Queda dar cuenta de -es. Echar mano de un pronombre o partícula enclítica podría ser una solución, ${ }^{47}$ pero todavía no podemos afirmar taxativamente que el celtibérico usase este procedimiento. ${ }^{48}$

En definitiva, no estamos en condiciones de poder dar una definición morfológica segura de esta forma.

\section{RECAPITULACIÓN}

A partir de una base originaria $* h_{2} e w-d^{h} e h_{1}^{-/ *} h_{2} e w-d^{h} h_{1}-\mathrm{o} * a w d^{h} e h_{1}^{-/ *} a w d^{h} h_{l^{-}}$, de significado 'conceder, otorgar, donar', en celtibérico podrían estar testimoniadas las siguientes formas:

audintum $<* a w-d^{\text {h }} e h_{l}-n t-\bar{o} m$ : genitivo del plural del participio de presente activo. auzeti $<* a w-d^{h} h,-e-t i$ : $3 .^{\mathrm{a}}$ singular presente de subjuntivo (temático) voz activa. auzanto $<* a w-d^{h} h_{l}-n t o: 3 .^{a}$ plural del aoristo radical atemático, indicativo, voz media.

auz, posible abreviatura de auzaz $<* a w-d^{h} h_{l}-t: 3 .^{\text {a }}$ singular del aoristo radical atemático, indicativo, voz activa.

auzimei: dativo singular de un tema en $-i$ o locativo singular de un tema en -o. En ambos casos la protoforma es *aw-d $d^{h} h_{1}-m e i$.

auzares: sin análisis morfológico claro, $i^{*} a u d^{h} h_{1}-r-e s$ ?

\section{NOTA FINAL}

No se nos escapa que si pudiésemos identificar uno de los elementos enclíticos aludidos en el final -um, entonces la forma audintum podría segmentarse como audintum. Aparecería así una forma personal, que podría entenderse, entre otras cosas, en un primer momento como una $3 .^{\mathrm{a}} \mathrm{p}$. pl. del presente de indicativo atemático con extensión del grado de la raíz desde el singular al plural: audint $<*$ au-d $d^{h} h_{1}-n t i$. Líneas arriba hemos hablado precisamente de la teórica $3{ }^{\mathrm{a}}$ p.sg. $* a u-d^{h} e h_{l}-t i>* a u-d^{h} \bar{e}-t i>$ *au-dt-ti, que se grafiaría $<$ auziti $>$ en signario paleohispánico, $<$ audit $(i)>$ en alfabeto latino. No en vano en la línea 7 del bronce se lee cabint. ${ }^{49}$ Sin embargo, no hemos sa-

${ }^{47}$ Así todavía, aunque con dudas, RuBio (2013: 310).

${ }^{48} C f$. Rubio (1998). De Bernardo (2009: 690 y 692) sostiene la existencia de un sujeto pronominal enclítico $-s$ en kombalkez [K.1.1, A1] $<*$-t-s y la terminación -tuz $<*$-tōd-s, pero son formas fácilmente explicables a partir de ${ }^{*}-t \mathrm{y}^{*}-d(c f$., por ejemplo, JoRDÁn 2004: 141 y ss.). Las otras dos palabras que aparecen en [K.0.14] con la misma terminación, es/ozeres [A-1] y tunares [B-1] no ayudan mucho a la identificación. Una vez más, vid. WKI ss. uu. para distintos análisis. Vid. en la nota 42 lo apuntado por PRÓSPER a propósito de esozeres y auzares; para la autora, en VILLAR y PRÓSPER (2005: 300), tunares también sería una tercera persona de aoristo del mismo verbo base, pero con distintos preverbios y elementos

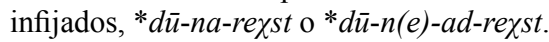

${ }^{49}$ Para posibles análisis de esta forma, vid. JordÁn (2014a). 
bido detectar ese elemento enclítico ni en celtibérico, ni en otra lengua indoeuropea. ${ }^{50}$ Teóricamente es fácilmente reconstruible, pero preferiríamos tener un punto de apoyo en la documentación.

\section{BibLIOGRAFÍA}

Beltrán, Francisco; Bienes, Juan José; Hernández, José Antonio, y Jordán, Carlos (2013), «El bronce celtibérico en alfabeto latino de Novallas (Zaragoza). Avance», PalHisp 13, 615-635.

de Bernardo, Patrizia (1999), Nominale Wortbildung des älteren Irischen. Stammbildung und Derivation, Tübingen.

- (2000), «Celib. karvo gortika 'amicitia favor', rita 'ofrenda', monima 'recuerdo' y los formularios de las inscripción celtibéricas», Veleia 17, 183-189.

- (2007), «Varietäten des Keltischen auf der Iberischen Halbinsel: Neue Evidenzen», en: H. Birkhan, (ed.), Kelten-Einfälle an der Donau. Akten des Vierten Symposiums deutschsprachiger Keltologinnen und Keltologen (Linz/Donau, 17.-21. Juli 2005), 149162, Wien: 149-162 .

- (2007a), «Cib. auzu 'haurio', auzeti 'haurit', auzanto 'hauriant': Water in the Botorrita bronzes and other inscriptions (K.0.8, 1.1, 1.3, 2.1, 5.1)», PalHisp 7, 55-69.

- (2007b), «Sull'origine delle sibilanti in celtiberico: Una modifica alla teoria di Francisco Villar», en P.-Y. LAmbert \& G.-J. Pinault (eds.), Gaulois et celtique continental, Genève: $182-188$.

- (2009), «La gramática celtibérica del Primer Bronce de Botorrita: nuevos resultados», PalHisp 9, 683-699.

- (2010), «La ley del 1. ${ }^{\text {er }}$ Bronce de Botorrita: uso agropecuario de un encinar sagrado», en F. Burillo (ed.), Ritos y Mitos. VI Simposio sobre celtíberos. Daroca (Zaragoza), 27-29 de noviembre de 2008, Zaragoza: 123-145.

— (2013), «El Tercer Bronce de Botorrita, veinte años después», PalHisp 13, 637-660.

Dunkel, Georg E. (2014), Lexikon der indogermanischen Partikeln und Pronominalstämme. Band 1: Einleitung, Terminologie, Lautgesetze, Adverbialendungen, Nominalsuffixe, Anhänge und Indices, Band 2: Lexikon, Heidelberg.

EsKA, Joseph F. (1996), «Further to the treatment of syllabic nasals in final position in protoCeltic», HS 109, 73-75.

JoRdÁn Cólera, Carlos (2004), Celtibérico, Zaragoza.

- (2005-2006), «Consideraciones paleo-epigráficas a propósito del Bronce Res», Kalathos 24-25, 475-486.

- (2014), «Chronica Epigraphica Celtiberica VIII», PalHisp 14, 289-324.

- (2014a), «La forma verbal cabint del bronce celtibérico de Novallas (Zaragoza)», Emerita LXXXII, 2, 327-343.

- (e.p.), «À propos de la valeur du $s$ diacrité dans les inscriptions celtibériques en alphabet latin», Études Celtiques, e.p.

${ }^{50}$ Para esta cuestión vid. Dunkel (2014). 
KloecKhorst, Alwin (2007), The Hittite inherited lexicon, Leiden [openaccess.leidenuniv. nl/ handle/1887/11996].

Lambert, Pierre-Yves (2002), Recueil des Inscriptions Gauloises, Volume II, fascicule 2, Paris.

- (2003), La langue gauloise, Paris.

LORRIO, Alberto J. y VeLAZA, Javier (2005), «La primera inscripción celtibérica sobre plomo», PalHisp 5, 1031-1048.

LuJán, Eugenio R. (2009), «Notas sobre algunas inscripciones paleohispánicas», PalHisp 9, 701-709.

McCone, Kim (1996), Towards a relative Chronology of ancient and medieval celtic Sound Change, Maynooth.

РокоRNY, Julius (1948-1959), Indogermanisches etymologisches Wörterbuch, Bern.

Prósper, Blanca María (2006), «Un paralelo léxico-sintáctico entre celtibérico y galo. La firma de alfarero gala AVOT y celtibérico auz», PalHisp 6, 151-163.

- (2007), Estudio lingüístico del plomo celtibérico de Iniesta, Salamanca.

- (2008), El bronce celtibérico de Botorrita I, Pisa - Roma.

Rix, Helmut (1976), Historische Grammatik des Griechischen. Laut- und Formenlehre, Darmstadt.

RIx, Helmut et alii (2001), Lexicon der indogermanischen Verben, Wiesbaden.

RuBio, Francisco (1996), «Tensiones paradigmáticas en el verbo celtibérico: auđeti, auđanto, auđares y otras formas emparentadas», Kalathos 15, 181-193.

- (1998), «En torno a los pronombres infijados en céltico continental», Veleia 15, 229-239.

Rubio, Francisco (1999a), «Das keltiberische Verb und der protokeltische Imperativ», HS 112, 1999, 106-121.

- (1999b): «El verbo celtibérico y el verbo protocéltico: Problemas y perspectivas», en F. VIllar \& F. Beltrán (eds.), Pueblos, Lenguas y Escrituras en la Hispania Prerromana. Actas del VII Coloquio sobre lenguas y culturas paleohispánicas (Zaragoza, 12 a 15 de Marzo de 1997), Salamanca: 619-634.

Schumacher, Stefan (2004), Die keltischen Primärverben, Innsbruck.

Untermann, Jürgen (1997), Monumenta Linguarum Hispanicarum. Band IV. Die Tartessischen, keltiberischen und lusitanischen Inschriften, Wiesbaden.

Villar, Francisco y Prósper, Blanca María (2005), Vascos, celtas e indoeuropeos. Genes y lenguas, Salamanca.

Villar, Francisco; DíAz, M. ${ }^{a}$ Antonia; Medrano, Manuel, y Jordán, Carlos (2001), El IV Bronce de Botorrita (Contrebia Belaisca): Arqueología y Lingüistica, Salamanca.

WodtKo, Dagmar (2000): Monumenta Linguarum Hispanicarum. Band V.1. Wörterbuch del ketliberischen Inschriften, Wiesbaden. 